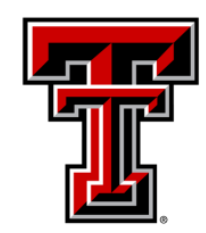

TEXAS TECH UNIVERSITY

Libraries"

\title{
REFLECTION ON MODERN METHODS: PLANNED MISSING DATA DESIGNS FOR EPIDEMIOLOGICAL RESEARCH
}

\section{The Texas Tech community has made this publication openly available. Please share how this access benefits you. Your story matters to us.}

\begin{tabular}{|l|l|}
\hline Citation & $\begin{array}{l}\text { Rioux, C., Lewin, A., Odejimi, O. A. \& Little, T. D. (2020). Reflection } \\
\text { on modern methods: Planned missing data designs for } \\
\text { epidemiological research. International Journal of Epidemiology. } \\
49(5), 1702-1711 . ~ \underline{\text { https://doi.org/10.1093/ije/dyaa042 }}\end{array}$ \\
\hline Citable Link & $\underline{\text { https://hdl.handle.net/2346/86921 }}$ \\
\hline Terms of Use & $\underline{\text { CC BY-NC }}$ \\
\hline
\end{tabular}


This is a pre-copyedited, author-produced version of an article accepted for publication in International Journal of Epidemiology following peer review.

Version of record: Rioux, C., Lewin, A., *Odejimi, O. A. \& Little, T. D. (2020). Reflection on modern methods: Planned missing data designs for epidemiological research. International Journal of Epidemiology. 49(5), 1702-1711. doi: 10.1093/ije/dyaa042.

Reflection on modern methods: Planned missing data designs for epidemiological research Charlie Rioux ${ }^{1 *}$, Antoine Lewin ${ }^{2,3}$, Omolola A. Odejimi ${ }^{1}$, Todd D. Little ${ }^{1}$

${ }^{1}$ Department of Educational Psychology and Leadership, College of Education, Texas Tech University, Box 41071, Lubbock TX, 79409-1071, United States

${ }^{2}$ Medical Affairs and Innovation, Héma-Québec, 4045 Boulevard Côte-Vertu, Montréal QC, H4R 2W7, Canada

${ }^{3}$ Faculty of Medicine and Health Science, University of Sherbrooke, 3001 12e Avenue N, Sherbrooke QC, J1H 5H3, Canada

Corresponding author: Charlie Rioux, Texas Tech University, Mail Stop 1071, Lubbock TX 79409-1071, tel.: 1-806-834-5651; email: charlie.rioux@ttu.edu. ORCID: 0000-0002-7943-0710 


\begin{abstract}
Taking advantage of the ability of modern missing data treatments in epidemiological research (e.g., multiple imputation) to recover power while avoiding bias in the presence of data that is missing completely at random, planned missing data designs allow researchers to deliberately incorporate missing data into a research design. A planned missing data design may be done by randomly assigning participants to have missing items in a questionnaire (multiform design) or missing occasions of measurement in a longitudinal study (wave-missing design), or by administering an expensive gold-standard measure to a random subset of participants while the whole sample is administered a cheaper measure (two-method design). Although not common in epidemiology, these designs have been recommended for decades by methodologists for their benefits - notably that data collection costs are minimized, and participant burden is reduced, which can increase validity. This paper describes the multiform, wave-missing and twomethod designs, including their benefits, their impact on bias and power, and other factors that must be taken into consideration when implementing them in an epidemiological study design. Keywords: Methods, Missing Data, Bias, Data Quality, Research Design, Questionnaire Design, Measurement
\end{abstract}




\section{Key messages}

- Planned missing data designs randomly assign values to be missing in a research design without introducing bias.

- Planned missing data designs decrease data collection costs, improve data quality and decreasing participant burden.

- The multiform design reduces the length of a questionnaire by randomly assigning missing questionnaire items.

- The wave-missing design randomly assigns participants to miss one or more data collection occasion(s).

- The two-method design randomly assigns participants to be administered a gold standard measure. 
In recent years, the availability of advanced statistical software has allowed epidemiological researchers to be able to ask increasingly complex questions about the interrelationships of health-related variables over time. One of the most useful aspects of modern statistical techniques is that they allow the presence of missing data. Missing data is a common problem in epidemiological research, which is associated with a potential for bias that needs to be addressed. While most researchers used to omit whole cases with any missing data (e.g., listwise deletion), missing data techniques embedded in newer statistical software have, in certain cases, enabled them to recover missing information, leading to reduced bias and increased statistical power. The availability and ease of use of missing data estimation techniques also make implementing a planned missing data designs possible. These designs allow researchers to collect incomplete data while ensuring that the missing data introduced is missing completely at random and, thereby, does not introduce any bias (see definition below). While this idea of deliberately incorporating missing data into a research design may sound perverse and has not been thoroughly discussed in epidemiology, planned missing data designs have been recommended for decades as an efficient way to decrease data collection costs, improve data quality and maintain statistical power while also decreasing test burden on participants and controlling the missingness pattern [1-4]. In the present paper, we first review missing data mechanisms and treatments, which are at the basis of the efficacy of a planned missing data design, as well as the state of missing data reporting in epidemiology. Second, three types of planned missing data designs that may be beneficial for epidemiologic research are introduced: multiform designs, wave-missing designs and two-method designs. 


\section{Missing data mechanisms and treatments}

Missing data can arise from three basic mechanisms known as missing completely at random (MCAR), missing at random (MAR), and missing not at random (MNAR) [5, 6]. If the missing data are MCAR, then the missing values are unrelated to the observed variables, missing values, and all unmeasured variables, meaning that the cases with missing data are not different to those with non-missing data. In the MCAR case, modern missing data estimation procedures (e.g., multiple imputation, full information maximum likelihood; see below) provide unbiased estimates and recover most of the power that would be lost by deleting cases with missing data. If the missing data are MAR, the missing values can be predicted by other variables in the observed data (e.g., if participants with a higher body mass index (BMI) tended to skip a particular question but not participants with a lower BMI, then the missing values would be predicted by BMI). In the MAR case, again, the missing data procedures can provide unbiased estimates and recover power, as long as the observed variables that are correlated with the missing values are included in the missing data treatment. The degree to which missing data treatments can help obtain unbiased results when the data is MAR depends on whether variables associated with the missing data are measured and on their strength of association. Finally, if the missing data are MNAR, then the missing values are predicted by missing variables (e.g., if the participants with a higher BMI tended to skip a question on weight in an assessment, and there are no other observed indicators of body composition). In the MNAR case, modern missing data treatments cannot recover the missing information, and thus estimates remain biased. It is thus important during the design phase of a study to plan to collect data on variables that are likely to predict missing values.

The two main recommended modern missing data estimation techniques are full 
information maximum likelihood (FIML) and multiple imputation [7, 8, 5, 9]. FIML uses all the available data (complete and incomplete) to produce the most accurate estimates of means, variances and covariances, parameter estimates and standard errors in a single step. FIML is implemented by most structural equation modeling [10] software, and it can be used for a wide range of simple and complex analyses. The second technique, multiple imputation, is more frequently used in epidemiological research. Multiple imputation is a two-step approach. In the first step, $m$ plausible values for each missing data point are imputed based on the information for the complete data. These values are substituted for the missing value to create $m$ complete datasets. In the second step, the analysis is carried on each dataset, and the results are combined across datasets. In other words, MI uses a model for the joint distribution of the data. With both methods, it is essential to include key auxiliary variables in the estimation procedure. Auxiliary variables are variables that may or may not be relevant to the theoretical model but are thought to be related to the presence of missing values (e.g., sex, ethnicity, age, education, socioeconomic status, comorbid diagnosis, BMI, medications, insurance access, etc. [11-14]) Measuring and including the right auxiliary variables can turn unpredictable missingness into predictable missingness, thus reducing bias in the analyses. Still, even when using recommended missing data treatments such as multiple imputation, results may be biased from MNAR data, and there are also instances when results will be biased when missing data is MAR [15-17]. Thus, despite the availability, ease of use, and effectiveness of modern missing data treatments, it remains important to make efforts in the design phase of a study to ensure a minimal rate of unplanned missing data. 
Review of missing data reporting

In order to give an idea of the state of missing data reporting and treatments in epidemiology, we reviewed articles published in 2018 in journals aimed at a general epidemiological audience with a 5-year impact factor > 5.0 [18]: the American Journal of Epidemiology, Epidemiology, the European Journal of Epidemiology, and the International Journal of Epidemiology. A sample of 100 studies that included questionnaire data for the predictors, outcomes, or covariates was randomly selected to be reviewed (see Supplementary Materials for more detailed methods and list of reviewed studies). Of those 100 articles, 87 mentioned missing data explicitly, with 66 reporting the percentage of data missing, which ranged from less than $0.1 \%$ to $66.5 \%$.

Furthermore, of the 87 articles mentioning missing data, only 11 discussed missing data mechanisms. Four of these 11 studies assumed a MAR mechanism without providing details on whether this assumption was tested, and seven tested predictors of the MAR mechanisms.

Of the 87 studies mentioning missing data, 77 gave details regarding missing data treatments. One study used FIML and 18 studies used multiple imputation. Regarding other missing data treatments, two studies used inverse probability weighting, four studies used single regression imputation, three studies used last observation carried forward, one study used mean substitution, two studies removed variables with missing data from their analytical plan, and 57 seven studies used deletion (used with other missing data treatments in 11 cases). Assuming that studies that do not mention missing data or their missing data treatment used complete case analysis, deletion-based missing data treatment was the most commonly used method (70\% of studies), followed by multiple imputation (18\% of studies).

Overall, this review suggests that there is still room for improvement regarding both missing data reporting and missing data treatment in epidemiology. $13 \%$ of studies did not report 
anything regarding missing data. Minimally, all authors should report the extent of missing data in their studies. If no missing data are present, this should be stated. Ideally, authors should also aim to use appropriate missing data treatments. Deletion-based techniques were the most commonly used. If deletion-based techniques are used in instances where they have been shown to yield valid results, this should be stated by the authors. This valid use of deletion methods, however, was most likely not the case for many studies using these techniques, which are known to frequently lead to biased results in addition to reducing power [5]. As explained above, modern missing data treatments such as multiple imputation can reduce bias and increase power when data is MCAR and MAR. These advantages of modern treatments are important because a majority of studies did not mention testing missing data mechanisms. Predictors of missingness need to be tested and included as auxiliary variables in the imputation model, otherwise, even if missingness is MAR, the results will be biased to the same extent that they would have been if the missingness was MNAR.

While proper missing data treatments minimize bias and maximize power when analyzing data with unplanned missing data, they also allow the implementation of planned missing data designs, which will be discussed next.

\section{Planned missing data designs}

Taking advantage of the potential of missing data treatments to recover power while avoiding any added bias for the portion of the data that is MCAR, planned missing data designs allow researchers to collect incomplete data by randomly assigning values to be missing in their research design. Planned missing data designs have been recommended because of their numerous benefits. First, these designs can reduce the length of a questionnaire or assessment, 
thereby reducing the burden on the participant, which can increase data quality, lower unplanned missingness due to skipped items, unfinished questionnaires, and/or attrition, and reduce data collection costs. Second, by reducing the length of assessments, planned missing data designs can allow more items to be assessed in a study without increasing the burden on the participant [19-21]. These advantages are particularly relevant to epidemiology where many studies rely on cohort studies with extensive, repeated assessments, and could benefit from methods to reduce participant burden while maintaining or increasing data quality. Because unplanned missing data is usually unavoidable, a reduction in unplanned missing data can result from the implementation of these designs [21], which could also improve data quality. As reviewed above, some epidemiological studies have a high missing data rate (above 50\%), and the benefit of reduced unplanned missingness may be especially important for studies where those higher missing data rates are expected (e.g., due to the design, population). The remainder of this paper introduces three planned missing data designs that could be beneficial for epidemiological research.

\section{Multiform designs}

The multiform design (also called split-questionnaire design, partial-questionnaire design and split-ballot design) reduces the length of a questionnaire by creating multiple forms that each contain a subset of the items to be assessed [22-25]. The simplest one of these designs is the three-form design, where all items are assigned to one of four sets (X, A, B, or C), which are then combined to create three forms (or questionnaire versions). As shown in Table 1A, each form is composed of the $\mathrm{X}$ block (sometimes called the "common" block) and two of the three remaining blocks. Participants are randomly assigned to one of the forms and some data is obtained for all pairs of items, which is important since it makes it possible to estimate all 
covariances.

Several considerations must be taken into account when constructing the item sets, notably regarding items assigned to the $\mathrm{X}$ set, which are the items answered by all participants. This set should include demographic variables as well as other key variables that may predict the MAR mechanism for unplanned missing data. These key variables can be identified through a literature search for the predictors of missingness in previous studies with populations and designs similar to the study in which the three-form design will be implemented. Some also advise that at least one item per scale should also be included, which usually is the most reliable item (e.g., with the highest factor loadings from a factor analysis, or the highest item-total correlations $[4,20])$. The rest of the items from each scale would be distributed across the remaining blocks (A, B or C) as sets of items. This method of distributing scale items across sets is associated with better results when examining associations between variables, with more efficient regression coefficients and correlations [26, 27], which is a benefit for most epidemiological studies. Another method would entail assigning whole scales to item sets, but is only efficient when a study's main objective is to examine scale structure (e.g., factor analysis $[26,27])$ It can also be beneficial to include single items (i.e., that are not part of a scale) in the $\mathrm{X}$ set, particularly low-base rate items such as suicide ideation or gating variables such as "are you pregnant?". The key to the three-form design is to randomly assign each person one of the three forms so that the missing items are missing completely at random.

Beyond the three-form design, the multiform design may include more item sets, which introduces more planned missingness and thus further reduces the length of the questionnaire. In all instances, each form would still include the $\mathrm{X}$ set and two additional sets, with all combinations of sets being included so that all covariances can be estimated (e.g., six-form 
design with five item sets, see Table 1B). The number of sets used may be influenced by the scales included in the study. Indeed, if the majority of the scales included in the study have a large number of items, they may be divided among more sets than if smaller scales are used [19]. Furthermore, when more sets are included, a lower proportion of participants will answer each pair of items. Thus, the number of sets should be limited unless sample is very large (about 40 participants per form is recommended as the minimum sample size [20]).

Multiform designs can be implemented both in cross-sectional and longitudinal studies. When implementing the design in a longitudinal study, different methods may be used to assign participants to forms over time (i.e., participants could have the same form at each measurement occasion, or a different form at each measurement occasion (recommended). One study found that when scales are distributed across sets (as recommended above for studies interested in associations between variables), the attribution of the same or different forms at each measurement occasion did not have an impact on efficiency (i.e., did not have an impact on the precision of the estimation of parameters [26]). Still, if repeated-testing effects are a concern with the variables included in the study, assigning different forms across time may be beneficial [20]. Furthermore, if scales were kept together within item sets (which, as mentioned above, is beneficial for factor analysis), assigning participants to different forms across measurement occasions was more efficient [26].

Such methods are suited for clinical questions where investigators wish to explore and understand an unknown phenomenon or test a hypothesis. For example, Varni et al. [28] published a study of parallel pediatric self-reports and parent proxy-report items investigating the individual item-level discrepancies between children aged 8-17 years and their parents using the PROMIS pediatric pain scale. Two hundred and ninety-three proxy report items from 10 content 
domains were administered to 1,548 parents of the 8- to 17-year-old children. To reduce respondent burden, a multiform design was used in which items were divided among nine forms, with each item appearing on three of the forms, and each parent was administered one of the nine forms [29, 28].

\section{Benefits and power considerations}

Simulation studies have shown that results with a multiform design are reliable and similar to results from complete data designs for both cross-sectional and longitudinal research [26, 30-32]. Participants perceive questionnaires from multiform designs to be shorter, less boring, and less repetitive [33], which may be particularly relevant for large cohort studies, which tend to administer long assessments to have data on a wide variety of variables, and are frequent in epidemiological research. Research has also shown that a reduction in participant fatigue and boredom obtained from a planned missing data design may lead to more valid data, stronger effects, and fewer skipped items [21]. Furthermore, multiform designs can reduce repeatedtesting effects when it is a concern in a study with multiple assessments, if participants answer different items at each assessment $[4,20]$. Still, statistical power needs to be considered since efficiency can be lower in the presence of missing data, meaning that standard errors are bigger and thus power declines. When using a multiform design, efficiency is lower when parameters are estimated using items from different sets. If scale items are spread across sets as recommended above, regression and correlation parameters estimated using these scales are efficient if the imputation is conducted at the item level, and the impact on power is minimized $[34,26,27]$.

A sample size of at least 115 was found to be sufficient for the implementation of the 
three-form design for the convergence of a one-time-point model and two-time-point model, and a sample size of 175 was found to be sufficient for a three-time-point mediation model

(convergence rate $>.9$, for three constructs, all measured at each time point, factor loadings $>.7$, within-time covariances .2 to .5 , autoregressive coefficients .4 to .9 , cross-lagged paths .1 to .4 , with 5\% unplanned missingness [35]). Similar to studies without planned missing data, the complexity of the analytic model and expected effect sizes must be taken into consideration when determining sample size. Monte Carlo simulations can be used for power analysis to determine the required sample size when implementing a three-form design, a method detailed in Schoemann et al. [36]. An advantage of this method is that it can estimate power while taking into consideration not only imposed planned missing data, but also expected levels of unplanned missing data (which, like effect sizes, can be estimated based on previous studies). These power analyses can be used for all planned missing data designs presented in this paper.

\section{Wave-missing designs}

The wave-missing (planned attrition) design randomly introduces planned wave nonresponse in a longitudinal study by assigning participants to miss one or more data collection occasion(s). Many patterns of missing data may be introduced (see Table 2) and researchers can tailor this design to maximize efficiency while reducing repeated measurement effects and costs. The best pattern of wave-missing data may differ depending on the research questions. Methodological research on the subject has been restricted to growth trajectories, for which concentrating the planned attrition and nonparticipation in the middle waves (see Table 2C) is associated with more efficient parameters $[37,38,30]$. These simulations suggest that the end points of a growth trajectory provide more information than the middle points for the estimation. Accordingly, for a 
spline function (i.e., two linear growth trajectories connected at a transition point), the estimation is more reliable when data is complete for the point of change and the planned wave-missing data is distributed among the other waves [39]. For studies examining growth trajectories that have hypothesized parameter values, an algorithm was developed to identify the most efficient pattern of planned missing data for the model [40]. Further methodological research is needed to identify whether wave-missing designs are efficient for longitudinal models other than growth trajectories.

A cross-sequential design is often used in epidemiology [e.g., 41, 42, 43] and can also be analyzed as a wave-missing design $[19,44]$. A cross-sequential design starts with a crosssectional design and participants are then followed longitudinally. For example, as shown in Table 3, cohorts of 50,51, and 52 year-olds at Wave 1 could be measured for four consecutive years until they are 53, 54, and 55 years old, respectively. This cross-sequential design can be transformed into a wave-missing accelerated longitudinal data design [44] where the youngest group is missing data at 54-55 years, the middle group is missing data at 50 and 55 years, and the oldest group is missing data at 50-51 years (see Table 3). In this example, a 6-year span is measured in just 4 years.An additional example of a wave-missing design in epidemiology could be applied to a time-lag model with variation in exposure times. For example, health effects associated with exposures to risk factors always occur with some delay and modeling temporal patterns of risk due to time-varying exposures is challenging. A design with time-lag exposure variation can be arrayed longitudinally, so that each lag is a potential data collection point, and each participant has data at the first time point plus one other time point (e.g., a participant with a 4-month time lag has complete data at time points 1 (baseline) and 5 (4 months), and missing data on time points 2 (1 month), 3 (2 months), 4 (3 months), and 6 (5 months)). In this way, 
complete two-time-point data are transformed into a planned missing data design with multitime-point data that can be analyzed as, for example, a growth curve model. This design is of particular interest in environmental epidemiology when investigating the short or long-term associations between exposures such as air pollution or whether variables and health outcomes such as mortality, myocardial infarction or disease-specific hospital admissions.

\section{Benefits and power considerations}

With similar benefits to multiform designs, wave-missing designs can reduce repeated-testing effects (if they are a concern for the variables included in the study), reduce participant burden, and dramatically reduce data collection costs. Despite these similar benefits, multiform designs applied longitudinally may be more efficient than wave-missing designs. Indeed, a study found that for longitudinal designs, multiform designs used at each occasion have a minimal impact on efficiency compared to wave-missing designs, which were associated with lower efficiency and more biased estimates and standard errors [30]. Conversely, cost savings can be greater with wave-missing designs, especially if assessments include laboratory or home visits and not only questionnaires, and thus the costs of lower efficiency need to be weighted with the benefits of the design [19], especially in a context of reduced research and public health funding [45, 46]. Monte Carlo power analyses can be used to determine whether a study will have the power to detect the hypothesized effects after implementing a wave-missing design [36]. Planned missing data designs may also be combined to maximize the benefits, for example with a multiform design for questionnaires and a wave-missing design for more costly laboratory visits or biological assessments. 
Two-method design

The two-method design is for the specific situation where researchers are faced with the choice between two measures: a gold standard that is more expensive, intrusive, time-dependent and/or time-consuming, and a biased measure that is inexpensive, non-intrusive, time-dependent and/or time-saving [23]. This dilemma may occur for many health-related variables commonly examined in epidemiology for which direct observation or biological assessments as well as selfreport measures are available, including, for example, smoking [47], stress [48], puberty [49], body composition [50], and physical activity [51]. Using the gold standard may lead to an underpowered study because its high cost results in a smaller sample size, but using the biased measure can reduce the validity of the study.

The two-method design introduces missing data by administering the biased measure to the whole sample of participants and administering the gold-standard measure to a random subsample of those participants. Therefore, this design allows researchers to get the best of both worlds: more power than if using only the gold-standard measure, and more validity than if using only the biased measure. The two-method design takes advantage of structural equation modeling to separate the latent bias from the latent construct. In other words, including the more valid gold-standard measure allows one to statistically control for the bias in the easily gathered measure. Importantly, the biased measure must be a systematically biased measure of the same construct that is assessed with the gold standard measure. Furthermore, because latent factors are used for this design, both the gold-standard and biased measures must include two or more indicators to distinguish the reliable variance from the unreliable variance and to parse the reliable variance into a bias construct and focal construct (i.e., the construct of interest) [23].

For example, as detailed in Graham et al. [23], although smoking self-reports are 
considered reliable in most circumstances, they may not be valid in instances where social desirability is greater, such as with adolescents or pregnant women. In such instances, the twomethod design may be used by having all participants fill out a self-report measure (with at least two indicators) and measuring biomarkers such as cotinine and carbon monoxide on a random subsample of participants. The two-method design would also be a good fit for epidemiological research on stress. Indeed, not only can stress be measured both through biomarkers (cortisol) and self-reports, but it has also been recommended to use both types of measures as stress is characterized by the co-occurrence of cortisol changes and the subjective appraisal related to a perceived inability to cope with unpredictable or uncontrollable situations $[52,48]$. Self-reported measures of stress, however, are systematically biased and objective cortisol measurements are more costly and demanding for participants. Thus, as with the smoking example, the two-method design could be used by having all participants fill out a self-reported stress questionnaire (with at least two indicators) and measuring cortisol (e.g., in hair and saliva) on a random subsample of participants. The stress variable would then be modeled with a latent factor approach (see Figure 1) where both the self-reports and cortisol measures load on a common factor (capturing the variance that is common across both measures), and the self-reports also load on a bias factor (capturing the variance that is shared only among the indicators of the self-reports after conditioning on the common factor). The resulting common factor would represent the common, unbiased variance between the self-reports and the cortisol measurements.

The two-method design can be implemented in longitudinal studies. On the one hand, if the bias in the biased measure is known to be equal over time, the gold standard may be measured at only one wave. On the other hand, if the bias changes over time, measuring the gold standard only once can result in biased results. Thus, when bias is unstable over time, the gold 
standard must be included at several measurement occasions for the results to be accurate and efficient. If the factor structure and stability of the bias over time are unknown, it may be safer to include the gold standard at every measurement occasion [53].

\section{Benefits and power considerations}

The key benefit of the two-method design is that it can maximize power and validity while managing costs and increasing study feasibility. When a construct is measured using the twomethod design and modeled using the latent variable approach described above before being included in a larger model, regression parameters were found to be (1) more valid than if only the biased measure was used, and (2) more efficient than if only the gold standard was used with a smaller sample (i.e., power is higher using the two-method design than using only the gold standard with a smaller sample [23]). These features are a key difference of the two-method design compared to the multiform and wave-missing designs. Indeed, while a reduction in efficiency must be factored into the power estimates when implementing the multiform and wave-missing designs, the two-method design increases power for small sample studies using gold standard measures or increases validity for large-sample studies using more biased measures.

The proportion of the sample that receives the gold standard is an important consideration. At a fixed cost, if more participants receive the expensive measure, sample size decreases along with efficiency, but if not enough participants receive the gold standard measure, the bias in the other measure becomes more difficult to estimate and efficiency also decreases. The ideal proportion of participants receiving the two measures depends on the reliability of the measures, the cost ratio between the two measures, and the effect sizes between the construct 
measured using the two-method design and other variables. Graham et al. discuss how to estimate the proportion of participants assessed with the expensive and inexpensive measures using these criteria [23] and Monte Carlo power analyses can also be used to determine sample size [36].

\section{Conclusion}

Missing data is ubiquitous in epidemiological research, with some studies having rates of missing data that are over 50\%. Although this missing data can bias results and reduce power, modern missing data treatments allow researchers to reduce these consequences of missing data. Still, as researchers can never be certain that unplanned missing data did not have an impact on their conclusions, prevention is key, and studies should be designed to minimize unplanned missing data and their impact on data analyzes, for example by making sure to collect potential auxiliary variables for the missing data treatments. Planned missing data designs can also be implemented and have been shown to have the potential to reduce unplanned missing data [21]. These designs also reduce participant burden, which can be beneficial for all studies, and especially beneficial for large epidemiological cohort studies with extensive, repeated assessments.

Methodologists have recommended planned missing data designs based on undeniably sound statistical theory, and the availability and ease of use of missing data treatments have made their implementation possible for applied researchers. Increasingly, methodological research provides sound recommendations for effective and valid use of these designs. As this research continues to grow, planned missing designs will undoubtedly become more common in many research fields, including epidemiology. If a planned missing data design is carefully 
planned for the specific needs of a study, both power and validity can be optimized. Power analyses taking planned and unplanned missing data into consideration should be conducted prior to data collection. Although missing data can decrease efficiency, reductions in cost obtained through the implementation of a planned missing data design can allow an increase in sample size that easily offsets the loss in power. Furthermore, through a reduction in participant burden (multiform design and wave-missing design) or use of a gold-standard measure (twomethod design), implementing a planned missing data design has the key advantage of increasing validity. Considering their many benefits discussed in the present article, planned missing data designs should definitely be utilized in the design of future epidemiological studies.

Funding: This work was supported by the Canadian Institutes for Health Research and the Fonds de Recherche du Québec - Santé through fellowships to [CR].

Conflicts of Interest: TDL owns and receives remuneration from Yhat Enterprises, which runs educational workshops such as Stats Camp (statscamp.org). The other authors declare that they have no conflict of interest. 


\section{References}

1. Shoemaker DM. Principles and procedures of multiple matrix sampling. Cambridge, MA:

Ballinger Publishing; 1973.

2. Sirotnik K. Introduction to Matrix Sampling for the Practitioner. In: Popham W, editor.

Evaluation in Education: Current Practice. Berkeley, CA: McCutchan; 1974. p. 451-529.

3. Palmer RF, Royall DR. Missing Data? Plan on It! J Am Geriatr Soc 2010; 58:S343-S8.

4. Little TD, Jorgensen TD, Lang KM, Moore EWG. On the Joys of Missing Data. J Pediatr Psychol 2014; 39:151-62.

5. Enders CK. Applied Missing Data Analysis. Methodology in the Social Sciences. New York, NY: The Guildford Press; 2010.

6. Seaman S, Galati J, Jackson D, Carlin J. What Is Meant by "Missing at Random"? Stat Sci $2013 ; 28: 257-68$.

7. Lang KM, Little TD. Principled Missing Data Treatments. Prev Sci 2018; 19:284-94.

8. Little RJA, Rubin DB. Statistical analysis with missing data. Hoboken, NJ: John Wiley \& Sons; 2014.

9. Sterne JAC, White IR, Carlin JB et al. Multiple imputation for missing data in epidemiological and clinical research: potential and pitfalls. British Medical Journal 2009; 339:9.

10. Beran TN, Violato C. Structural equation modeling in medical research: a primer. BMC Research Notes 2010; 3:267-77.

11. Clark TG, Altman DG. Developing a prognostic model in the presence of missing data: an ovarian cancer case study. J Clin Epidemiol 2003; 56:28-37.

12. Fabricatore AN, Wadden TA, Moore RH et al. Attrition from randomized controlled trials of pharmacological weight loss agents: a systematic review and analysis. Obes Rev 2009; 10:333- 
41.

13. Robinson L, Adair P, Coffey M, Harris R, Burnside G. Identifying the participant characteristics that predict recruitment and retention of participants to randomised controlled trials involving children: a systematic review. Trials 2016; 17:17.

14. Stuart EA, Azur M, Frangakis C, Leaf P. Multiple Imputation With Large Data Sets: A Case Study of the Children's Mental Health Initiative. Am J Epidemiol 2009; 169:1133-9.

15. Kristman VL, Manno M, Cote P. Methods to account for attrition in longitudinal data: Do they work? A simulation study. Eur J Epidemiol 2005; 20:657-62.

16. Lewin A, Brondeel R, Benmarhnia T, Thomas F, Chaix B. Attrition Bias Related to Missing Outcome Data: A Longitudinal Simulation Study. Epidemiology 2018; 29:87-95.

17. Twisk J, de Vente W. Attrition in longitudinal studies: How to deal with missing data. J Clin Epidemiol 2002; 55:329-37.

18. Clarivate Analytics. 2017 Journal Citation Reports2018.

19. Little TD, Rhemtulla M. Planned Missing Data Designs for Developmental Researchers. Child Develop Perspect 2013; 7:199-204.

20. Rhemtulla M, Hancock GR. Planned Missing Data Designs in Educational Psychology Research. Educ Psychol 2016; 51:305-16.

21. Harel O, Stratton J, Aseltine R. Designed missingness to better estimate efficacy of behavioral studies-application to suicide prevention trials. Journal of Medical Statistics and Informatics $2015 ; 3: 2$.

22. Graham JW, Hofer SM, MacKinnon DP. Maximizing the usefulness of data obtained with planned missing value patterns: An application of maximum likelihood procedures. Multivariate Behav Res 1996; 31:197-218. 
23. Graham JW, Taylor BJ, Olchowski AE, Cumsille PE. Planned missing data designs in psychological research. Psychol Methods 2006; 11:323-43.

24. Wacholder S, Carroll RJ, Pee D, Gail MH. The partial questionnaire design for case-control studies. Stat Med 1994; 13:623-34.

25. Raghunathan TE, Grizzle JE. A Split Questionnaire Survey Design. J Am Stat Assoc 1995; 90:54-63.

26. Jorgensen TD, Rhemtulla M, Schoemann A, McPherson B, Wu W, Little TD. Optimal assignment methods in three-form planned missing data designs for longitudinal panel studies. Int J Behav Dev 2014; 38:397-410.

27. Rhemtulla M, Savalei V, Little TD. On the asymptotic relative efficiency of planned missingness designs Psychometrika 2016; 81:60-89.

28. Varni JW, Thissen D, Stucky BD et al. Item-level informant discrepancies between children and their parents on the PROMISA (R) pediatric scales. Qual Life Res 2015; 24:1921-37.

29. Irwin DE, Gross HE, Stucky BD et al. Development of six PROMIS pediatrics proxy-report item banks. Health Qual Life Outcomes 2012; 10:13.

30. Rhemtulla M, Jia F, Wu W, Little TD. Planned missing designs to optimize the efficiency of latent growth parameter estimates. Int J Behav Dev 2014; 38:423-34.

31. Smits N, Vorst HCM. Reducing the length of questionnaires through structurally incomplete designs: An illustration. Learn Individ Differ 2007; 17:25-34.

32. Swain MS. The effects of a planned missingness design on examinee motivation and psychometric quality [Doctoral dissertation]. Harrisonburg, VA: James Madison University; 2015.

33. Adiguzel F, Wedel M. Split Questionnaire Design for Massive Surveys. J Mark Res 2008; 
45:608-17.

34. Gottschall AC, West SG, Enders CK. A Comparison of Item-Level and Scale-Level Multiple Imputation for Questionnaire Batteries. Multivariate Behav Res 2012; 47:1-25.

35. Jia F, Moore EWG, Kinai R, Crowe KS, Schoemann AM, Little TD. Planned missing data designs with small sample sizes: How small is too small? Int J Behav Dev 2014; 38:435-52.

36. Schoemann AM, Miller P, Pornprasertmanit S, Wu W. Using Monte Carlo simulations to determine power and sample size for planned missing designs. Int J Behav Dev 2014; 38:471-9.

37. Graham JW, Taylor BJ, Cumsille PE. Planned missing-data designs in analysis of change. In: Collins PE, Sayer AG, editors. New methods for the analysis of change. Washington, DC: American Psychological Association; 2001. p. 335-53.

38. Mistley SA, Enders CK. Planned missing data designs for developmental research. In: Laursen B, Little TD, Card NA, editors. Handbook of developmental research methods. New York, NY: The Guildford Press; 2012. p. 742-54.

39. Hogue CM, Pornprasertmanit S, Fry MD, Rhemtulla M, Little TD. Planned missing data designs for spline growth models in salivary cortisol research. Meas Phys Educ Exerc Sci 2013; $17: 310-25$.

40. Wu W, Jia F, Rhemtulla M, Little TD. Search for efficient complete and planned missing data designs for analysis of change. Behav Res Methods 2016; 48:1047-61.

41. Clifford SA, Davies S, Wake M et al. Child Health CheckPoint: cohort summary and methodology of a physical health and biospecimen module for the Longitudinal Study of Australian Children. BMJ Open 2019; 9:3-22.

42. Hasselhorn HM, Peter R, Rauch A et al. Cohort profile: The lidA Cohort Study-a German Cohort Study on Work, Age, Health and Work Participation. Int J Epidemiol 2014; 43:1736-49. 
43. Thurber KA, Banks E, Banwell C, Lsic Team. Cohort Profile: Footprints in Time, the Australian Longitudinal Study of Indigenous Children. Int J Epidemiol 2015; 44:789-800.

44. Little TD. Longitudinal Structural Equation Modeling. Methodology in the Social Sciences. New York, NY: The Guilford Press; 2013.

45. Atkinson RD, Foote C. U.S. Funding for University Research Continues to Slide.

Washington, DC: Information Technology \& Innovation Foundation 2019.

46. McKillop M, Ilakkuvan V. The impact of chronic underfunding of America's publich health system: Trends, risk, and recommendations. Washington, DC: Trust for America's Health 2019. 47. Stevens KR, Munoz LR. Cigarette smoking: Evidence to guide measurement. Res Nurs Health 2004; 27:281-92.

48. Weckesser LJ, Dietz F, Schmidt K, Grass J, Kirschbaum C, Miller R. The psychometric properties and temporal dynamics of subjective stress, retrospectively assessed by different informants and questionnaires, and hair cortisol concentrations. Sci Rep 2019; 9:12.

49. Dorn LD, Biro FM. Puberty and Its Measurement: A Decade in Review. J Res Adolesc 2011; $21: 180-95$.

50. Toomey CM, Cremona A, Hughes K, Norton C, Jakeman P. A Review of Body Composition Measurement in the Assessment of Health. Top Clin Nutr 2015; 30:16-32.

51. Dowd KP, Szeklicki R, Minetto MA et al. A systematic literature review of reviews on techniques for physical activity measurement in adults: a DEDIPAC study. Int J Behav Nutr Phys Act 2018; 15:33.

52. Koolhaas JM, Bartolomucci A, Buwalda B et al. Stress revisited: A critical evaluation of the stress concept. Neurosci Biobehav Rev 2011; 35:1291-301.

53. Garnier-Villarreal M, Rhemtulla M, Little TD. Two-method planned missing designs for 
longitudinal research. Int J Behav Dev 2014; 38:411-22. 


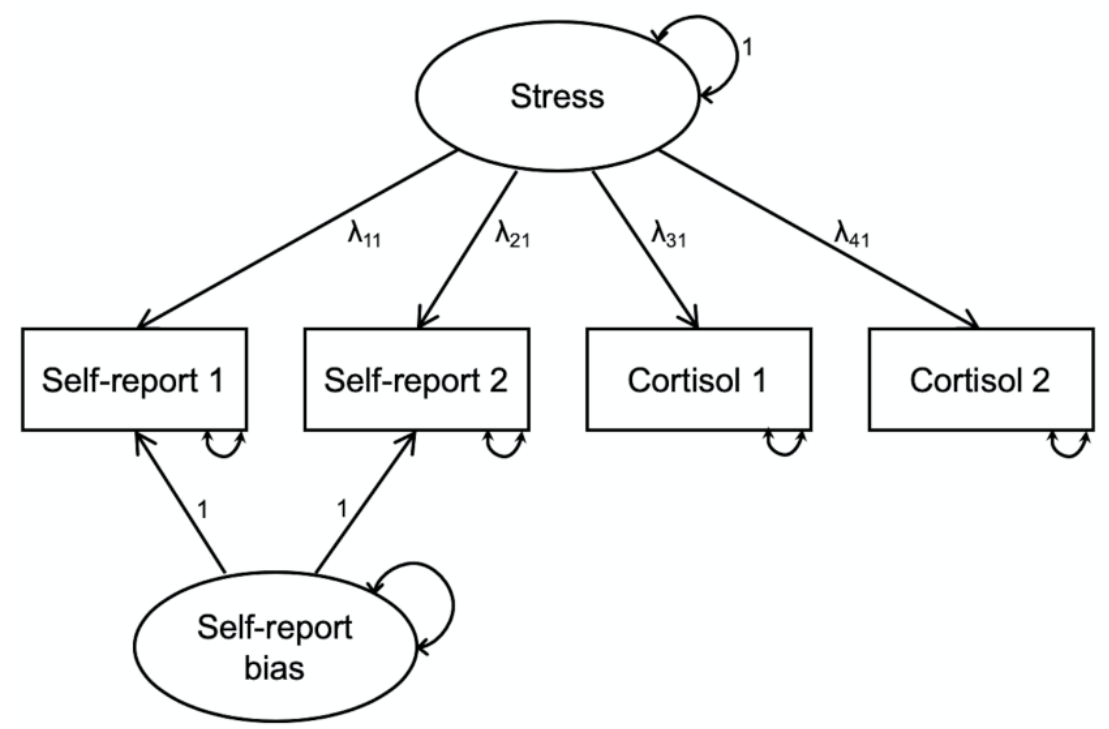

Figure 1 Path diagram illustrating the latent variable modeling of the two-method design. k 1/4 Estimated loading of an indicator on the construct. 
Table 1 Two multiform designs

\begin{tabular}{lccccc}
\hline \multicolumn{7}{l}{ A. Three-form design } & & \\
\hline Form & Set X & Set A & Set B & Set C \\
\hline 1 & $\checkmark$ & $\checkmark$ & $\checkmark$ & - \\
2 & $\checkmark$ & $\checkmark$ & - & $\checkmark$ \\
3 & $\checkmark$ & - & & $\checkmark$ & $\checkmark$ \\
\hline \hline \multicolumn{7}{l}{ B. Six-form design } & & & \\
\hline \multicolumn{7}{l}{ Form } & Sex X & Set A & Set B & Set C & Set D \\
\hline 1 & $\checkmark$ & $\checkmark$ & $\checkmark$ & - & - \\
2 & $\checkmark$ & $\checkmark$ & - & $\checkmark$ & - \\
3 & $\checkmark$ & - & $\checkmark$ & $\checkmark$ & - \\
4 & $\checkmark$ & $\checkmark$ & - & - & $\checkmark$ \\
5 & $\checkmark$ & - & $\checkmark$ & - & $\checkmark$ \\
6 & $\checkmark$ & - & - & $\checkmark$ & $\checkmark$ \\
\hline
\end{tabular}

$\checkmark$ item set is administered; - item set is omitted. 
Table 2 Three examples of planned wave-missing designs

\begin{tabular}{lllllll}
\hline \multicolumn{7}{l}{ A. Monotone Decreasing } \\
\hline Group & $\mathrm{W}_{1}$ & $\mathrm{~W}_{2}$ & $\mathrm{~W}_{3}$ & $\mathrm{~W}_{4}$ & $\mathrm{~W}_{5}$ & $\mathrm{~W}_{6}$ \\
\hline 1 & $\checkmark$ & $\checkmark$ & $\checkmark$ & $\checkmark$ & $\checkmark$ & $\checkmark$ \\
2 & $\checkmark$ & $\checkmark$ & $\checkmark$ & $\checkmark$ & $\checkmark$ & - \\
3 & $\checkmark$ & $\checkmark$ & $\checkmark$ & $\checkmark$ & - & - \\
4 & $\checkmark$ & $\checkmark$ & $\checkmark$ & - & - & - \\
5 & $\checkmark$ & $\checkmark$ & - & - & - & - \\
\hline \hline
\end{tabular}

B. One wave missing

\begin{tabular}{lllllll}
\hline Group & $\mathrm{W}_{1}$ & $\mathrm{~W}_{2}$ & $\mathrm{~W}_{3}$ & $\mathrm{~W}_{4}$ & $\mathrm{~W}_{5}$ & $\mathrm{~W}_{6}$ \\
\hline 1 & $\checkmark$ & $\checkmark$ & $\checkmark$ & $\checkmark$ & $\checkmark$ & - \\
2 & $\checkmark$ & $\checkmark$ & $\checkmark$ & $\checkmark$ & - & $\checkmark$ \\
3 & $\checkmark$ & $\checkmark$ & $\checkmark$ & - & $\checkmark$ & $\checkmark$ \\
4 & $\checkmark$ & $\checkmark$ & - & $\checkmark$ & $\checkmark$ & $\checkmark$ \\
5 & $\checkmark$ & - & $\checkmark$ & $\checkmark$ & $\checkmark$ & $\checkmark$ \\
\hline \hline
\end{tabular}

C. Middle waves missing

\begin{tabular}{lllllll}
\hline Group & $\mathrm{W}_{1}$ & $\mathrm{~W}_{2}$ & $\mathrm{~W}_{3}$ & $\mathrm{~W}_{4}$ & $\mathrm{~W}_{5}$ & $\mathrm{~W}_{6}$ \\
\hline 1 & $\checkmark$ & $\checkmark$ & $\checkmark$ & - & - & $\checkmark$ \\
2 & $\checkmark$ & $\checkmark$ & - & $\checkmark$ & - & $\checkmark$ \\
3 & $\checkmark$ & - & $\checkmark$ & $\checkmark$ & - & $\checkmark$ \\
4 & $\checkmark$ & $\checkmark$ & - & - & $\checkmark$ & $\checkmark$ \\
5 & $\checkmark$ & - & $\checkmark$ & - & $\checkmark$ & $\checkmark$ \\
6 & $\checkmark$ & - & - & $\checkmark$ & $\checkmark$ & $\checkmark$ \\
\hline
\end{tabular}

$\checkmark$ item set is administered; - item set is omitted; W $=$ Wave of assessment (i.e., measurement occasion) 
Table 3 Example of a cross-sequential design transformed into a wave-missing accelerated longitudinal data design

\begin{tabular}{|c|c|c|c|c|c|c|}
\hline & \multicolumn{6}{|c|}{ Cohort-sequential design } \\
\hline Cohort initial age & $\mathrm{W} 1$ & $\mathrm{~W} 2$ & W3 & W4 & & \\
\hline 50 years & 50 & 51 & 52 & 53 & & \\
\hline 51 years & 51 & 52 & 53 & 54 & & \\
\hline \multirow[t]{3}{*}{52 years } & 52 & 53 & 54 & 55 & & \\
\hline & \multicolumn{6}{|c|}{$\begin{array}{l}\text { Transformation into the wave-missing accelerated longitudinal design } \\
\text { Full span of ages covered and wave of assessment for each cohort }\end{array}$} \\
\hline & 50 years & 51 years & 52 years & 53 years & 54 years & 55 years \\
\hline 50 years & $\mathrm{W} 1$ & W2 & W3 & W4 & & \\
\hline 51 years & & W1 & W2 & W3 & W4 & \\
\hline 52 years & & & W1 & W2 & W3 & W4 \\
\hline
\end{tabular}

$\mathrm{W}=$ Wave of assessment. Adapted from Little (2013) [44]. 